\title{
Phlegmonous gastritis after endoscopic submucosal dissection for early gastric cancer
}

\author{
Hironari Ajibe, Hiroyuki Osawa, Mitsuyo Yoshizawa, Hironori Yamamoto, Kiichi Satoh, \\ Koji Koinuma, Kazue Morishima, Yoshinori Hosoya, Yoshikazu Yasuda and Kentaro Sugano
}

\begin{abstract}
A 74-year-old man with diabetic nephropathy developed epigastric pain and high fever after endoscopic submucosal dissection (ESD) for early gastric cancer. Gastroscopy, endoscopic ultrasonography and computed tomography showed ulceration with a purulent lake, thickened entire gastric mucosal layers suggesting focal abscess formation, leading to the diagnosis of phlegmonous gastritis. He underwent total gastrectomy as an emergency. Histological findings of the resected specimen showed severe inflammatory cell infiltration and multiple focal abscess formation spreading to the entire gastric wall. In patients with poorer general conditions, phlegmonous gastritis should be considered as a serious complication after ESD, indicating a requirement of antibiotic prophylaxis.
\end{abstract}

Keywords: Phlegmonous gastritis, severe complication, early gastric cancer, endoscopic submucosal dissection

\section{Introduction}

Gastric cancer is one of the most prevalent malignancies in the world. Recent developments in endoscopic procedures can improve patient care in early gastric cancer [Ohkuwa et al. 2001; Yamamoto et al. 2001; Yamamoto et al. 2002; Miyamoto et al. 2002]. Endoscopic submucosal dissection (ESD) has been developed as one of the new therapeutic modalities for early gastric cancer and is considered to be a relatively safe method with low invasiveness [Miyamoto et al. 2002; Yamamoto et al. 2002]. Although bleeding and perforation are the most frequent complications associated with ESD, these can be adequately managed without surgical intervention in most cases and other serious complications are considered to be rare.

Phlegmonous gastritis is an uncommon, often fatal condition characterized by suppurative bacterial infection of the stomach [Kim et al. 2005]. It may arise from a local or a disseminated haematogenous infection and may involve either a portion of the stomach (localized type) or the entire stomach (diffuse type). Here we report a rare case with phlegmonous gastritis, developed after ESD for early gastric cancer. The patient was treated initially with antibiotic therapy but required surgery on emergency. Phlegmonous gastritis should be recognized as a severe complication after ESD and in cases with immune compromised states we should take precautions against its development.

\section{Case report}

A 74-year-old man with diabetic nephropathy was introduced to our hospital to treat early gastric cancer. He had a depressed-type early gastric cancer in the antrum. Pathological diagnosis of the lesion was papillary adenocarcinoma (Figure 1a). The flexible spectral imaging colour enhancement (FICE) named by the Japanese Society of Spectral Imaging for endoscopy in June 2008 showed a clear demarcation line between the cancerous lesion and the surrounding area, (Figures 1b and 1c) [Osawa et al. 2008] and the endoscopic ultrasonography (EUS) showed a cancer lesion confined within the mucosal layer, fulfilling the extended criteria for ESD [Gotoda et al. 2006]. Therefore, the patient was scheduled to undergo ESD.
Therapeutic Advances in Gastroenterology (2008) 1(2) 91-95 DOI: $10.1177 /$ $1756283 \times 08095746$ C) SAGE Publications 2008 Los Angeles, London, New Delhi and Singapore

Correspondence to: Hiroyuki Osawa Department of Internal Medicine, Division of Gastroenterology, Jichi Medical University, 3311-1 Yakushiji, Shimotsuke. Tochigi 329-0498, Japan osawåajichi.ac.jp

Hironari Ajibe Mitsuyo Yoshizawa Hironori Yamamoto Kiichi Satoh Kentaro Sugano Department of Internal Medicine, Division of Gastroenterology Jichi Medical University, Tochigi, Japan

Koji Koinuma Kazue Morishima Yoshinori Hosoya Yoshikazu Yasuda Department of Surgery, Jichi Medical University, Tochigi, Japan 


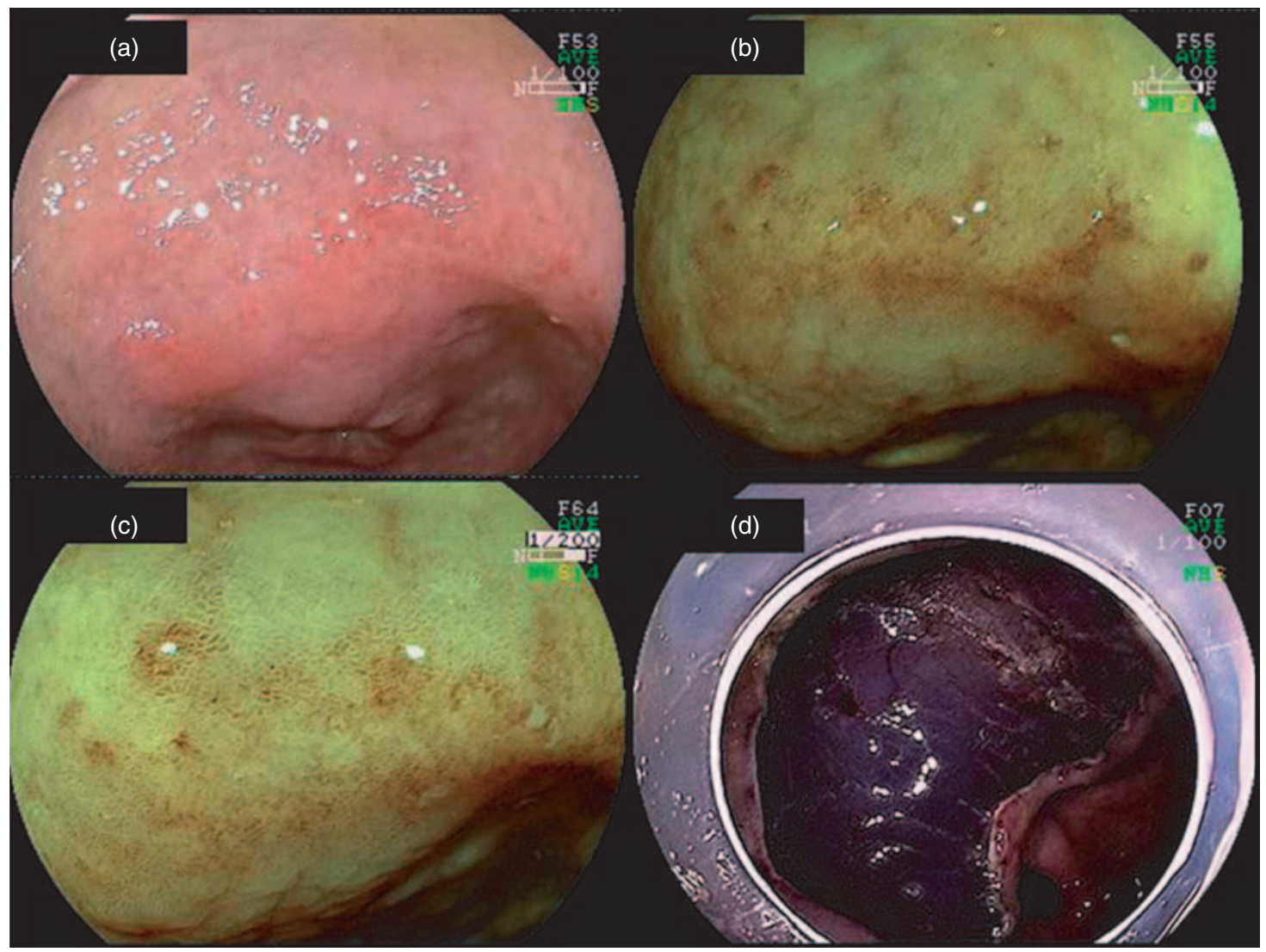

Figure 1. Conventional endoscopic image (a) and FICE image (b) of the slightly depressed lesion in the antrum. FICE showed a clear demarcation line between the cancerous lesion and the surrounding area. A 40-fold magnified FICE image (c) showed an irregular microstructural pattern, suggestive of gastric cancer. The ulceration after ESD using sodium hyaluronate solution (d).

The patient had been having haemodialysis for 15 years because of diabetic nephropathy. He had a moderate anaemia with $9.7 \mathrm{~g} / \mathrm{dl}$ of haemoglobin concentration, a lower level of platelet count and white blood cells, $111,000 / \mu 1$ and $3400 / \mu 1$, respectively, but levels of coagulation factors were normal. Blood chemistry data on admission were normal except for renal functions. $\mathrm{He}$ had undergone ESD using sodium hyaluronate solution without any technical problems (Figure 1d). The size of the mucosa resected en bloc was $59 \times 27 \mathrm{~mm}$ in diameter including $40 \times 11 \mathrm{~mm}$ of cancer lesion. Five hours later, he complained of epigastric pain and on the next day he developed high fever of $39^{\circ} \mathrm{C}$ and vomited a small amount of blood. Endoscopic examination showed large coagulation and protruded vessels in the ulceration after ESD, which was treated with endoscopic coagulation therapy. He began to eat soft meals from two days after ESD, but the epigastric pain and signs of rebound tenderness continued. He had high fever of $39^{\circ} \mathrm{C}$ again from the five days after ESD and higher levels of CRP $(30 \mathrm{mg} / \mathrm{ml})$. Computed tomography (CT) showed a thickened gastric wall along the antral area, suspicious of phlegmonous gastritis (Figure 2d). The endoscopic examination on the seven days after ESD showed oedematous mucosa and ulceration with a yellowish purulent lake. (Figure 2a and b). EUS showed a diffusely thickened gastric wall extending to the muscle layers, suspicious of abscess formation (Figure 2c).

Citrobacter freundii, Enterobacter cloacae and $\alpha$-Streptococcus were cultured from his gastric secretion. The epigastric pain and high fever then transiently disappeared after antibiotic treatment with flomoxef (FMOX $0.5 \mathrm{~g} /$ day), but relapsed 10 days later. CT showed a thickened gastric wall extending to subserosa with multiple low density areas suspicious of focal abscess formation and impending perforation (Figure 3c). The endoscopic examination revealed diffuse ulceration with a massive purulent lake, strongly 

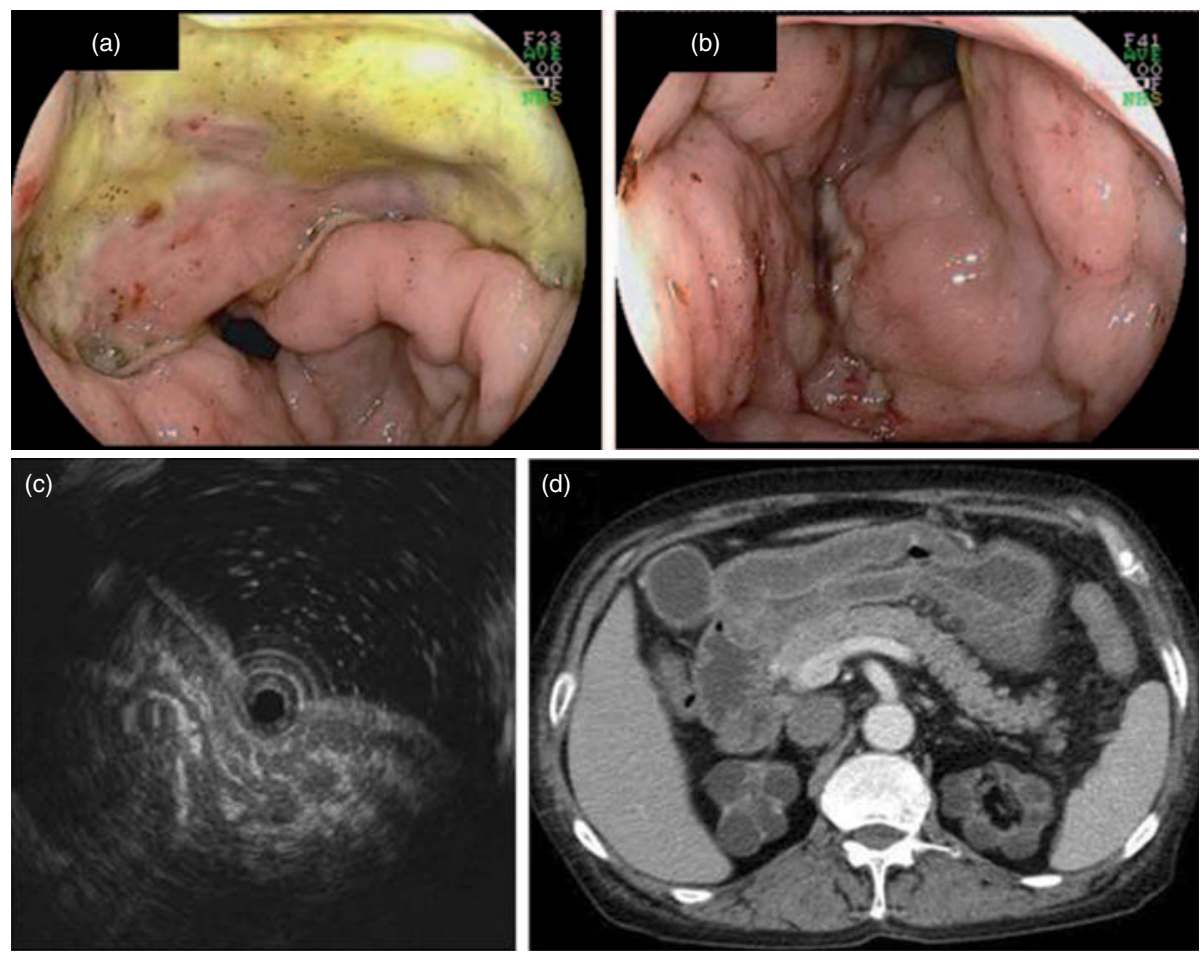

Figure 2. The ulceration on the seven days after ESD was enlarged and covered by yellowish purulent mucus (a). The gastric mucosa was diffusely oedematous and new ulceration was visualized in the middle body (b). EUS showed a thickened and irregular hyperehoic third layer corresponding to the submucosal layer on the greater curvature (c). Abdominal CT revealed a diffusely thickened gastric wall, suggesting phlegmonous gastritis (d).
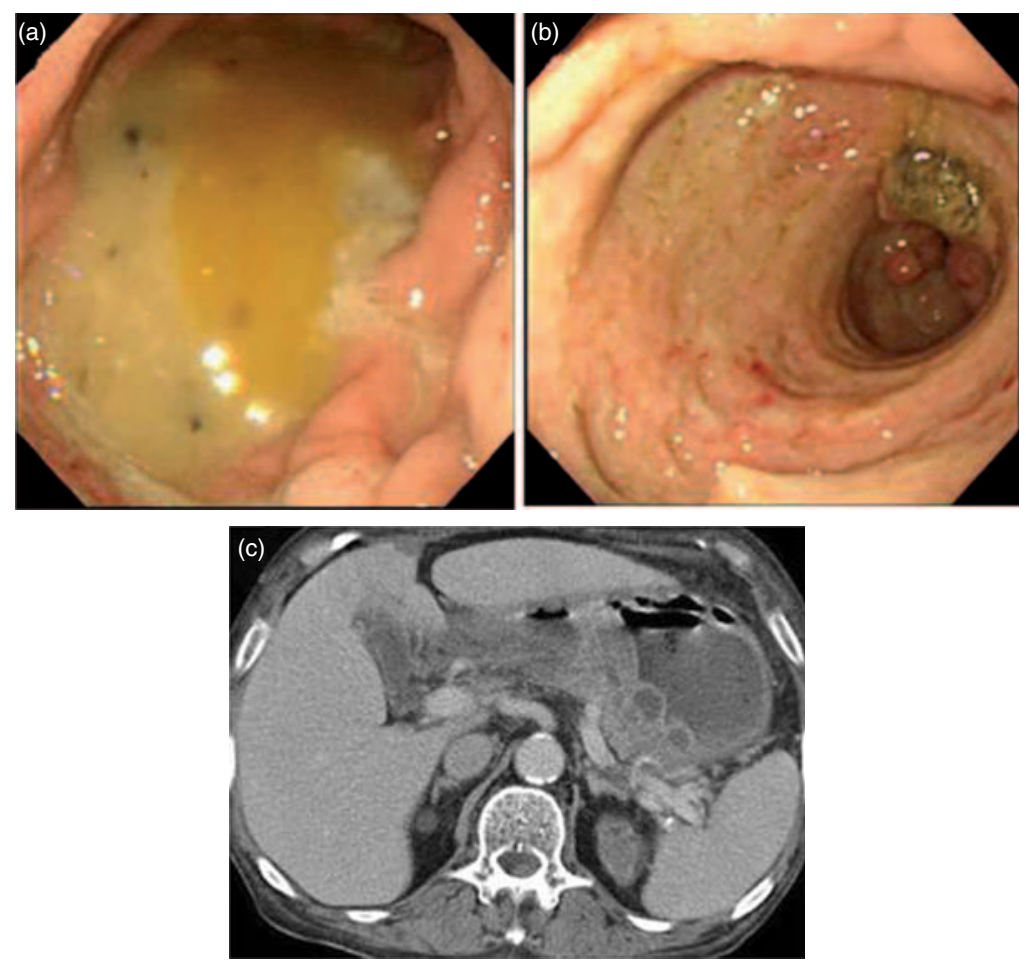

Figure 3. Endoscopic examination showed the massive purulent lake in the stomach (a) and the diffuse ulceration extended to the proximal part of the stomach (b) on the 17 days after ESD. CT showed a thickened gastric wall with multiple low density areas suggestive of focal abscess formation approximating submucosa (c). 

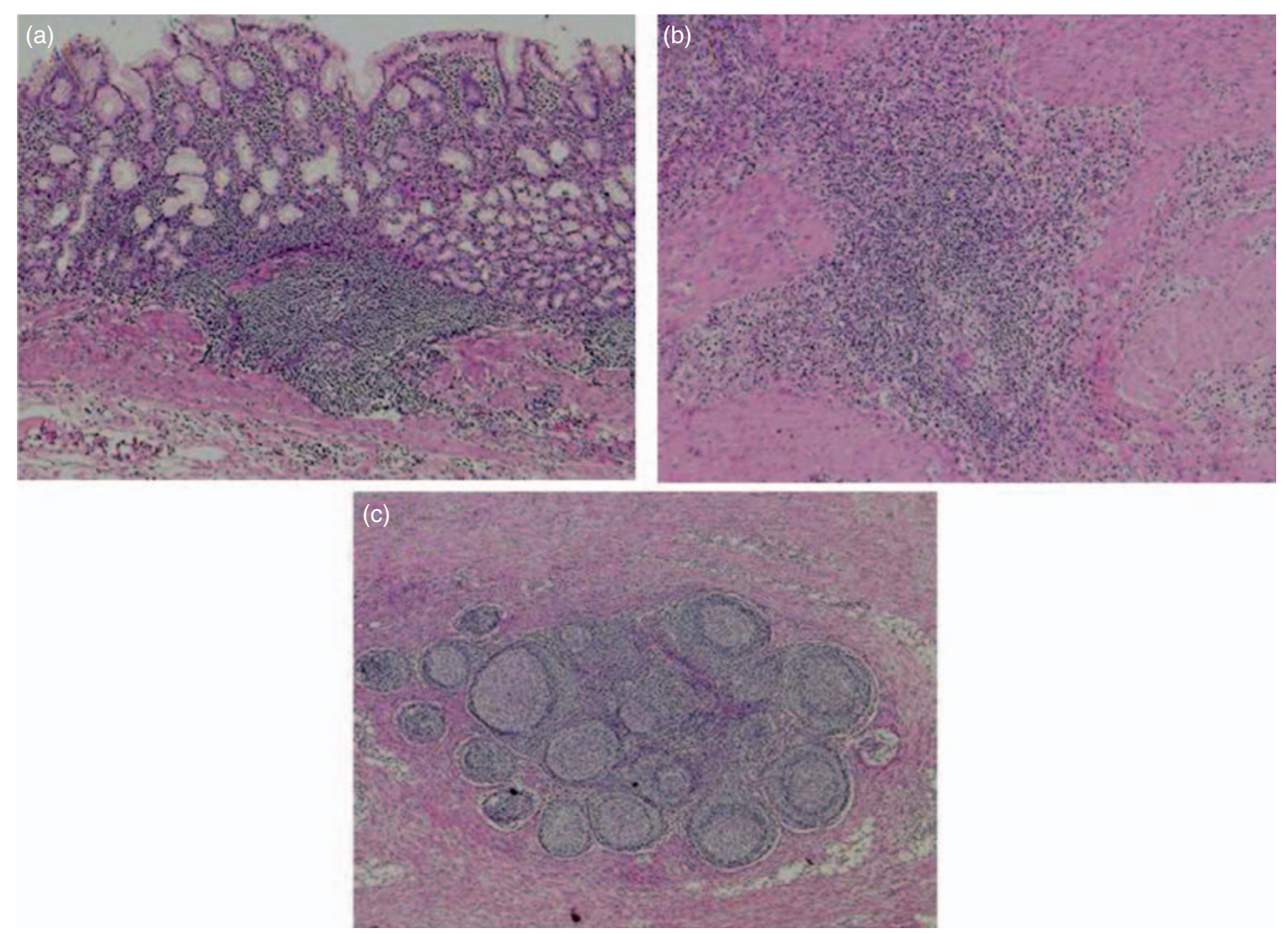

Figure 4. Postoperative histological views (hematoxylin and eosin) of the mucosa (a), submucosa and upper muscular layer (b), and serosa (c), showing acute suppurative gastritis with multifocal abscess formation and infiltration of inflammatory cells in all layers of the stomach.

indicating a high risk of perforation and sepsis in agreement with the CT findings (Figure 3a and b). Thus, he underwent total gastrectomy on emergency. Histological findings of the resected specimen were consistent with phlegmonous gastritis with severe inflammatory cell infiltration and multiple focal abscess formation in the entire gastric wall, but no perforation. (Figure $4 \mathrm{a}-\mathrm{c}$ ) $\mathrm{He}$ recovered uneventfully after gastrectomy.

\section{Discussion}

In this report, we present a case with phlegmonous gastritis as a rare and severe complication after ESD. This is the first report of phlegmonous gastritis after ESD for early gastric cancer. Phlegmonous gastritis, although a rare condition, is a submucosal bacterial infection with characteristic macroscopic and histological findings. Endoscopy, EUS and CT are useful in the diagnosis of phlegmonous gastritis [Danny et al. 2000; Sood et al. 2000; Iwakiri et al. 1999]. The most common pathogens responsible for phlegmonous gastritis are Streptococcus spp, Staphylococcus spp,
Escherichia coli, Haemophilus influenza, Proteus, and Clostridia [Miller AI et al. 1975; Schultz et al. 1996; Stephenson et al. 1970]. Citrobacter freundii isolated from gastric mucus in the present case has never been reported before. This bacterium is a member of the family Enterobacteriaceae and is often the cause of significant opportunistic infections. C. freundii has been associated with neonatal meningitis and brain abscess, [Joaquin et al. 1991] and could be responsible for phlegmonous gastritis in our case. The natural history of phlegmonous gastritis is that it deteriorates gradually leading to a life-threatening condition; the mortality rate of patients medically treated for the focal type is $17 \%$, and for the diffuse type is $60 \%$ [Kim et al. 2005]. Therefore, the combination of early radical gastric resection and vigorous antibiotic therapy appears to be the treatment of choice in phlegmonous gastritis [Lee et al. 2005; Yokota et al. 2005]. Such a clinical course was consistent with our case.

In previous literature, the causative factors of phlegmonous gastritis have included predisposing 
factors such as severe alcoholism, gastric haemorrhage, achlorhydria, and mucosal injury, acquired immune deficiency syndrome, and also after endoscopy [Hommel et al. 2007; Iwakiri et al. 1999; Kim et al. 2005; Lee et al. 2005]. Our patient had diabetic nephropathy and chronic severe atrophic gastritis that might induce lower gastric acid production. In patients with such underlying conditions, we must be more cautious of the risk of phlegmonous gastritis after ESD. However it is noteworthy that even without such apparent underlying conditions, phlegmonous gastritis develops after endoscopic procedures. Bron et al. reported on a patient who developed phlegmonous gastritis after endoscopic snare biopsy [Bron et al. 1977]. Another case of phlegmonous gastritis after endoscopic mucosal resection (EMR) of smaller size that required total gastrectomy has been reported [Lee et al. 2005]. Since post-ESD ulceration tends to be larger than post-EMR ulceration, the present case with diabetic nephropathy might be a high risk for phlegmonous gastritis. With the advent of the ESD technique, early gastric cancer with a diameter greater than $30 \mathrm{~mm}$ has been treated in Japan [Yamamoto et al. 2001]. In patients with poorer general conditions, administration of proper antibiotics should be considered to prevent phlegmonous gastritis after ESD.

\section{Conflict of interest statement \\ None declared.}

\section{References}

Bron, B.A., Deyle, P., Pelloni, S., Krejs, G.J., Siebenmann, R.E. and Blum, A.L. (1977) Phlegmonous gastritis diagnosed by endoscopic snare biopsy. Am F Dig Dis 22: 729-733.

Danny, C., Kevin, M., Paul, S., McGrath, M.D., Paul, S., Jowell, M.D. et al. (2000) Phlegmonous gastritis: successful treatment with antibiotics and resolution documented by EUS.

Gastrointestinal Endoscopy 52: 793-795.

Gotoda, T., Yamamoto, H. and Soetikno, R.M. (2006)

Endoscopic submucosal dissection of early gastric cancer. F Gastroenterol 41: 929-942.

Hommel, S., Savoye, G., Lorenceau- Salvale, C., Costaglioli, B., Baron, F., Le Pessot, F. et al. (2007) Phlegmonous gastritis in a 32 -week pregnant woman managed by conservative surgical treatment and antibiotics. Dig Dis Sci 52: 1042-1046.

Iwakiri, Y., Kabemura, T., Yasuda, D., Okabe, H., Soejima, A., Miyagahara, T. et al. (1999) A case of acute phlegmonous gastritis successfully treated with antibiotics. F Clin Gastroenterol 28: 175-177.

Joaquin, A., Khan, S., Russel, N. and al Fayez, N. (1991) Neonatal meningitis and bilateral cerebellar abscesses due to Citrobacter freundii. Pediatr Neurosurg 17: 23-24.

Kim, G.Y., Ward, J., Henessey, B., Peji, J., Godell, C., Desta, H. et al. (2005) Phlegmonous gastritis: case report and review. Gastrointest Endosc 61: 168-174.

Lee, B.S., Kim, S.M., Seong, J.K., Kim, S.H., Jeong, H.Y., Lee, H.Y. et al. (2005) Phlegmonous gastritis after endoscopic mucosal resection. Endoscopy 37: 490-493.

Miller, A.I., Smith, B. and Rogers, A.I. (1975) Phlegmonous gastritis. Gastroenterology 68: 231-238.

Miyamoto, S., Muto, M., Hamamoto, Y., Boku, N., Ohtsu, A. and Baba, S. (2002) A new technique for endoscopic mucosal resection with an insulated-tip electrosurgical knife improves the completeness of resection of intramucosal gastric neoplasms.

Gastrointest Endosc 55: 576-581.

Ohkuwa, M., Hosokawa, K., Boku, N., Ohtu, A., Tajiri, H. and Yoshida, S. (2001) New endoscopic treatment for intramucosal gastric tumors using an insulated-tip diathermic knife. Endoscopy 33: 221-226.

Osawa, H., Yoshizawa, M., Yamamoto, H., Kita, H., Satoh, K., Ohnishi, H. et al. (2008) Optimal band imaging system can facilitate detection of changes in depressed-type early gastric cancer. Gastrointest Endosc 67: 226-234.

Schultz, M.J., van der Hulst, R.W. and Tytgat, G.N. (1996) Acute phlegmonous gastritis. Gastrointest Endosc 44: 80-83.

Sood, B.P., Kalra, N. and Suri, S. (2000) CT features of acute phlegmonous gastritis. Clin Imaging 24: $287-288$.

Stephenson Jr, S.E., Yasrebi, H., Rhatigan, R. and Woodward, E.R. (1970) Acute phlegmasia of the stomach. Am Surg 36: 225-231.

Yamamoto, H., Kawata, H., Sunada, K., Satoh, K., Kaneko, Y., Ido, K. et al. (2002) Success rate of curative endoscopic mucosal resection with circumferential mucosal incision assisted by submucosal injection of sodium hyaluronate. Gastrointest Endosc 56: 507-512.

Yamamoto, H., Sekine, Y., Higashizawa, T., Kihira, K., Kaneko, Y., Hosoya, Y. et al. (2001) Successful en bloc resection of a large superficial gastric cancer by using sodium hyaluronate and electrocautery incision forceps. Gastrointest Endosc 54: 629-632.

Yokota, T., Yamaki, T., Yashima, R., Yamada, Y., Narushima, Y., Kikuchi, S. et al. (2005)

Emergency operation for phlegmonous gastritis.

Ups f Med Sci 110: 237-240.
Visit SAGE journals online http://tag.sagepub.com

(9SAGEJOURNALS Online 